\title{
ORGANISATIONAL FACTORS AFFECTING INTER-ORGANISATIONAL KNOWLEDGE TRANSFER IN THE NEW ZEALAND STATE SECTOR: A CASE STUDY
}

Jocelyn Cranefield and Pak Yoong

\begin{abstract}
This paper reports part of a larger research project that identified the key factors that impacted on knowledge transfer in the Pathfinder project, a two-year project undertaken by a group of New Zealand State Sector organisations, based around introducing a new strategic knowledge framework, Managing for Outcomes (MfO). Interviews were undertaken with participants from seven public sector organisations. An emergent model for inter-organisational knowledge transfer was developed from the data. The model comprises six stages: Engaging, Defining, Seeking, Articulating, Integrating, and Disseminating. This paper specifically describes the organisational factors that have affected inter-organisational knowledge transfer during each of the stages. The implications for research and practice are outlined.
\end{abstract}

Keywords: Knowledge transfer, network organisation 


\section{INTRODUCTION}

The transfer of knowledge within organisations is widely considered to be a key to effective knowledge management, and an enabler of the innovation that is necessary to enhance organisational capability (Earl, 1998; Nonaka, 1998). However, successful knowledge transfer is considered difficult (Gorgoglione, 2003; Harada, 2003; Nonaka, 1998); and the sharing of knowledge is perceived as risking a loss of power by some individuals (Scmetz, 2002). For these reasons, organisations need to actively work to create a knowledge sharing culture, and to facilitate practices and processes that promote effective knowledge transfer. In order to do this, it is necessary for them to understand the factors affecting knowledge transfer that apply to their organisational context.

The majority of research into knowledge transfer has been focused at the organisational level, but recent studies have uncovered the benefits of knowledge transfer and/or knowledge sharing in inter-organisational contexts including strategic alliances (Reid et al., 2001; Rolland \& Chauvel, 2000), business clusters (Yoong, 2003), value chains (Rolland \& Chauvel, 2000); and informal networks and communities of practice (N. Allen \& Leeuwen, 2003; Wenger, 2000)(Wenger, 2002; Allen \& Leewen, 2003).

In the context of the State Sector, however, the topic of inter-organisational knowledge sharing, and indeed the broader area of knowledge management, has received remarkably little attention in the research literature (Pardo, Cresswell, Zhang \& Thompson, 2001; Taylor \& Wright, 2004). This is a significant gap, given that since the 1990's there has been a move towards greater accountability in the State Sector in New Zealand and other countries (such as England, Canada, and Australia), bringing it closer to the private sector (Betancourt, 1997).

This paper reports part of a larger research project that identified the key factors that impacted on inter-organisational knowledge transfer in the Pathfinder project, a two-year project undertaken by a group of New Zealand State Sector organisations, based around introducing a new strategic knowledge framework, Managing for Outcomes (MfO) ${ }^{1}$. The paper begins by providing a brief summary of relevant literature. The second section describes the case research method used in the study. The third section describes the key organisational factors affecting inter-organisational knowledge transfer and the final section identifies implications for research and practice.

\footnotetext{
${ }^{1}$ Managing for Outcomes is an outcome-based approach to planning, management and reporting in the Public Service (Advisory Group for the Review of Centre, 2001). Its aim is to improve the performance of the Public Service and it requires organisations to adopt a strategic and outcome-focused approach to planning, management and reporting while focusing on delivering outputs
} 


\section{RELEVANT LITERATURE}

\subsection{Knowledge Transfer}

Davenport and Prusak (1998, p. 5) define knowledge as "a fluid mix of framed experience, values, contextual information, and expert insight that provides a framework for evaluating and incorporating new experiences and information". This typifies the views of KM theorists in that it places the value of knowledge in its use, and not its existence per se (Snowden, 1999).

KM theory adopts two knowledge categories first established by the philosopher Polanyi (1958, Cited in Davidson, 2002) - explicit knowledge (knowledge that can be codified), and tacit knowledge ('know-how' type knowledge that is not readily codified). Hedlund (1994) proposes a model where the two types of knowledge (tacit and explicit) reside at four levels of an organisation's social hierarchy: individual, group, organisation, and inter-organisational.

A key theme in KM literature is that because tacit knowledge is difficult to capture, organisations need to actively encourage its transfer through social acts of knowledge sharing (Earl, 1998; Nonaka, 1998).

Knowledge transfer is an area of KM concerned with the movement of knowledge across the boundaries created by specialised knowledge domains (Carlile \& Rebentisch, 2003). The importance of knowledge transfer for successful organisational innovation is a recurring theme in the literature. Knowledge can only be valuable if it is appropriate, accurate and accessible to its users, so its effective transfer requires a framework of systems, methods and procedures, and an appropriate organisational culture (Karlsen \& Gottschalk, 2004).

Two aspects affecting the success of knowledge transfer are the transparency of an organisation (the extent to which it is open to communication) and absorptive capacity (its readiness and ability to recognise the value of new knowledge, and to absorb and apply it), (Rolland et al., 2003). Absorptive capacity can be facilitated by shared cognitive bases (similar mental models and backgrounds, ibid).

According to Gorgoglione (2003), two key cognitive processes are involved in knowledge transfer at the individual level: the "upstream" act of codification (the process through which knowledge is represented in forms such as language, models and images) and the "downstream" act of interpretation (understanding the codified knowledge). Fundamental to both cognitive processes is the selection and organisation of information, which is affected by cognitive characteristics of individuals, and their background, goals, values and beliefs. Difficulties may arise in selecting the right code, motivating people to share knowledge, making knowledge accessible, and interpreting the coded information correctly. If the goal of knowledge transfer is training, consistency between cognitive systems is seen as beneficial, but if the goal is innovation, different cognitive systems are considered beneficial. This is because innovation requires 'fertilization', leading to the production of diverging behaviours 
(ibid). Gorgoglione's argument suggests that high absorptive capacity (Rolland et al., 2003) may not always be beneficial to knowledge transfer.

Various KM authors agree that knowledge sharing is a critical stage in knowledge transfer (Nonaka, 1998; Scmetz, 2002), but Liebowitz (2002) goes even further, seeing KM as undertaken largely for the purpose of creating a knowledge sharing culture, fostering collaboration and communication, and so in turn enhancing organisational innovation.

\subsection{Inter-organisational knowledge transfer}

Inter-organisational knowledge transfer is considered to be a key to innovation in strategic alliances(Reid et al., 2001; Rolland \& Chauvel, 2000), business clusters (Yoong, 2003), and value chains (Rolland \& Chauvel, 2000). In addition, inter-organisational knowledge sharing can occur within informal networks (N. Allen \& Leeuwen, 2003) and communities of practice (CoPs), groups of individuals who interact on a regular basis and are bound together by a shared interest area and by the value they place on shared learning in that area (Wenger, 2000). CoPs typically evolve informally, but Hildreth et al. (1998)contend that formally mandated groups can also work well as CoPs.

Despite the range of inter-organisational structures described above, much of the recent literature appears to be based within two contexts: strategic alliances and business clusters. It is possible that these settings have attracted interest due to the unique challenges faced by participating organisations: they commonly exist in an environment that involves both competition and co-operation, known as "co-opetition" (Loebecke et al., 1999).

Firms typically engage in alliances in order to gain competitive advantage by generating new knowledge (Reid et al., 2001). They may also be motivated by a desire to protect their knowledge assets, block rivals, or access networks (ibid). Waits (2000 Cited in Yoong, P. and Molina, M. (2003)) contends that cluster-based collaboration adds value to businesses through the activities of co-informing, co-learning, co-marketing, co-purchasing, and co-building economic foundations. Lee and Al-Hawamdeh (2002) and Husted and Michailova (2003), claim that knowledge sharing is an essentially unnatural act, so an analysis of the anticipated costs and benefits of an alliance informs the decision to participate.

Based on a review of public corporate announcements, Rolland and Chauvel (2000) found that "the basic intent of an alliance is seldom expressed as a desire to acquire knowledge, but rather as the development of new competencies or the acquisition of new market niches" (p.235).

\subsection{Inter-organisational knowledge transfer in the State sector}

There is remarkably little literature based around inter-organisational knowledge transfer in a government or State Sector environment, and no models appear to exist for knowledge transfer in this context. This is significant given the recent trend (since the 1990's) in New 
Zealand and other countries (notably England and Australia), towards greater accountability in the State Sector, bringing it closer to the private sector (Betancourt, 1997), and creating an environment in which there is an indisputable need for innovation.

New Zealand has the distinction of having recently taken "the most radical approach (amongst OECD countries) to government reform by placing the emphasis on results and outputs rather than on control of inputs" and "by holding public managers responsible for the outputs as if they were private enterprises" (ibid, p. 21). The Report of the Advisory Group on the Review of the Centre (Advisory Group for the Review of Centre, 2001) called for major changes in the New Zealand State Sector, noting that "significant shifts in emphasis are needed to better respond to the needs of the future" (p.4). Three areas for attention are noted: a need for better integrated, citizen-focused service delivery; a need to address fragmentation and improve alignment; and a need for a "culture shift" including more dynamism and innovation, in order to enhance the sector.

Despite the signalled need for change, there is little literature that specifically indicates the value of inter-organisational knowledge transfer in the New Zealand State Sector. The literature focuses instead around the topic of innovation, usually with no explicit acknowledgement of the role that knowledge transfer plays in innovation.

\section{METHOD}

The case research method was used in this study. Case research method is considered useful in situations such as this study, when the study is exploring a contemporary event, where control of subjects or events is not necessary and where there is not already an established theoretical base (Benbasat et al., 1987; Yin, 1994). Case research method can also shed light on a phenomenon (process, event, person or object of interest) and has been noted for three purposes; to produce detailed descriptions of a phenomenon, develop possible explanations of it, or evaluate the phenomenon. Case research method is considered useful when a natural setting is needed and when the study is exploring a contemporary event, where control of subjects or events is not necessary and where there is not already an established theoretical base (Benbasat et al., 1987). It is considered an appropriate research method for organisational studies because of the way it uses direct observation and systematic interviewing to gather data, and in particular when "how" or "why" questions are being posed (Yin, 1994) .

Seven participants were interviewed in this study. The participants were managers or senior policy analysts who had been a representative for their organisation in the Pathfinder project. Each interview took about one hour. The audio-tapes were immediately transcribed so that key points were noted and themes and ideas teased out prior to the next interview. All interview notes and transcriptions were returned to the participants for checking and validation. 
Data analysis was conducted simultaneously with data collection. Open keyword coding was conducted by assigning codes and labels in the margins of the transcriptions and they were often a summary of a section of data that was considered to be important to the study. Basically, the data analysis involved reviewing the interview material, drawing common themes and summarising the findings of the interviews.

\section{RESULTS}

In this section, we will provide a description of the context of the study, the Pathfinder Project, the Stages of Inter-organisational Knowledge Transfer, and the key factors affecting inter-organisational knowledge transfer during each stage.

\section{The Pathfinder Project}

The context for this study is the Pathfinder Project (Pathfinder), a collaborative State Sector project undertaken in New Zealand from 2001-2003 that has previously been identified by government as a successful example of inter-agency collaboration (Wright \& de Joux, 2003). Pathfinder aimed to develop outcomes-based management systems and share lessons learned, with the over-arching goal of raising sector performance by shifting management focus from the delivery of outputs towards the achievement of measurable results (Pathfinder, 2003). Participating individuals were charged with developing the emerging concept of Managing for Outcomes (MfO), constructing associated models, tools and procedures, and introducing $\mathrm{MfO}$ practice into their respective organisations.

\section{The Stages of Inter-organisational Knowledge Transfer}

In order to represent the combined and common experiences of knowledge transfer reported by the seven participants, a six-stage model of knowledge transfer from the inter-organisational project group to the participating organisations has been developed (see Figure 1). The model comprises six key stages: (1) Engaging, (2) Defining, (3) Seeking, (4) Articulating, (5) Integrating, and (6) Disseminating. The model is based on syntheses of experiences as reported by the research participants, so does not necessarily closely represent the experience of any single organisation. While it shows the stages sequentially, in reality there was an iterative approach to knowledge transfer as new ideas were taken up in succession and in light of previous learning. The model summarises this overall process. The next sections provide a brief description of each stage. 


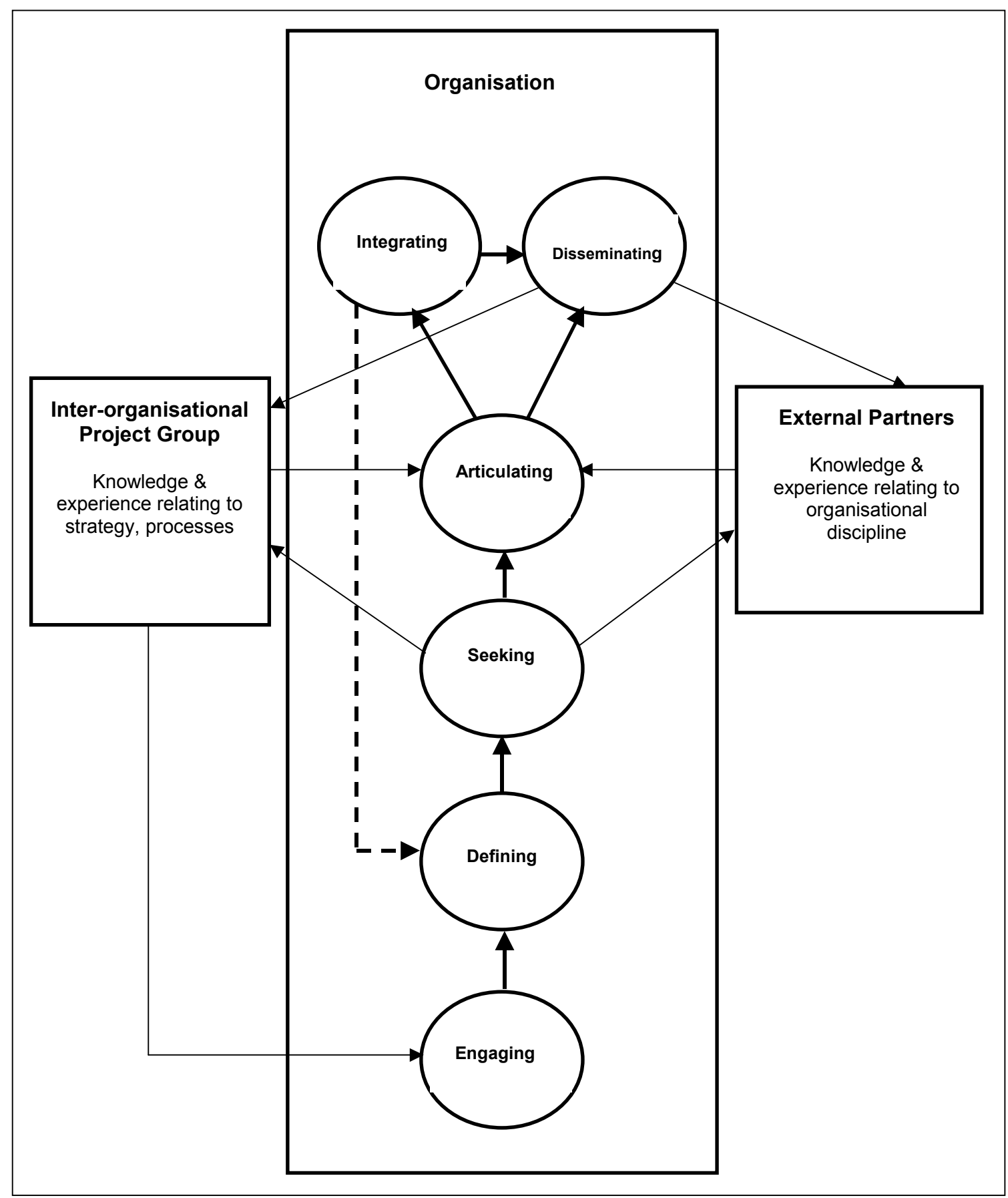

Figure 1. Model of Knowledge Transfer: Inter-organisational Project Group to Organisation

\section{Stage 1: Engaging}

In the first stage, Engaging, each organisation determined the level and nature of its engagement in the project, setting in place an initial framework for the process of knowledge transfer. The Engaging stage can be subdivided into two parts: (a) Recognising the challenge, and (b) Decision-making about the level of engagement. 
Recognising the challenge

At the outset of this stage, each organisation determined the degree of challenge that was posed by the project and its objective of introducing a new strategic management knowledge framework. The key factor affecting the degree of perceived challenge was the extent of each organisation's prior familiarity with the key concepts underlying the new knowledge framework: outcomes and MfO. Initial perceptions about the project and motivations for participating, were closely linked with their experience, and varied widely.

Decision-making about the level of involvement

During the decision-making part of the Engaging stage, organisations formalised the framework for engagement, setting up project working groups, defining objectives and a reporting framework, and assigning staff resources. The extent of reported engagement by participating organisations varied considerably and was strongly correlated with the degree of perceived "fit" between the new knowledge and an organisation's discipline area.

Several participants also described a requirement to act as an organisational flag-bearer at this stage of the project. This involved ensuring that their organisation was seen by central sponsoring agencies as contributing to the overall project, and that its role in determining its own agenda for the project was accepted. The Engaging stage therefore provided an important "window of opportunity" for organisations to influence their own direction.

\section{Key factors affecting knowledge transfer during the Engaging stage}

Analysis of the interviews showed the following factors impacted on knowledge transfer during the Engaging stage:

\section{(a) Prior experience with the new knowledge framework}

Prior organisational experience in working with the concept of outcomes was closely linked with the degree of perceived challenge (as has been discussed above) and strongly correlated with the level of engagement. Familiarity with the MfO knowledge framework appears to have resulted in a climate of readiness and confidence, leading to a higher level of engagement.

Participants from organisations that were already familiar with MfO reported strong engagement and a sense of having had a head start. Three of the four participants from organisations, which undertook a medium, or high level of project engagement also reported prior familiarity with MfO. Two organisations with a high degree of familiarity with MfO elected a narrow focused, but well-resourced project, supported by a range of communication mechanisms (medium level of engagement)). On the other hand, the participant who reported the lowest level of organisational familiarity with MfO also reported a low level of engagement:

"The (lack of) experience of the organisation meant that perhaps we didn't or couldn't contribute as much as those people in (other) organisations." 
(b) Fit with existing organisational objectives

The level of engagement was also strongly linked with the degree of perceived fit between the project and existing organisational objectives, being highest in organisations that viewed the project as a way of moving forward along an already established course and lowest where it was reported as being an additional demand of little immediate relevance.

\section{(c) Fit with traditional discipline area}

The degree of perceived fit between the MfO knowledge framework and the organisation's discipline area was also correlated with the level of engagement. Two interviewees noted a strong degree of fit with their organisation's historical discipline and its way of framing strategic knowledge.

"We'd been doing it for some years...(Our organisation's purpose) is the ultimate outcome Over time we have... pushed...new resource into programmes which we knew would be effective... So it got called outcomes management."

Both of these organisations undertook medium levels of engagement, with dedicated project teams working on focused areas where MfO work had not previously been undertaken.

Conversely, three participants noted that the concept of MfO was problematic in terms of its fit with their organisations' social science-orientated practice. They noted that although social science disciplines are generally focused on achieving outcomes, it is very difficult to link successful outcomes to specific organisational actions:

"If you've got an outcome chain, for example, if we carry out our activities and that results in a buoyant economy where every child is supported adequately and every person has a place in the community, how much can (we) actually affect that end-of-chain outcome? Because there's all the influence of other agencies, there's the influence of the world economy..(The difficulty is) working out where our spheres of influence end and where we can attribute our influence to outcomes."

\section{(d) CEO support and expectations}

The role of a supporting CEO, and their expectation of results from the project, was noted by several participants as affecting the level of engagement.

\section{(e) Competing issues}

In two cases, concurrent, competing work issues appear to have contributed to a low level of engagement. A heavy organisational workload was described as a problem by these two participants, both of whom also reported low levels of organisational engagement. In one case, this was due to a temporary event, a sudden increase in workload resulting from the government's response to world events of 9/11/2001. In the other case, the participant described a generally chaotic work environment, with the frequent introduction of new ideas:

"(The organisation) is far too flexible. It takes on a new idea every week...lt's chaotic. We leap from strategy to strategy." 
The same participant described having applied a screening process to gauge the project's relative value, leading to an early decision to restrict the level of engagement:

"This department has lots of things going on at once and in terms of providing information to people....we're very careful about what adds value...And (this project) wasn't going to set the world on fire."

This is notable, given the level of stakeholder expectation on the part of central agencies. However, it appears that a combination of factors was at work in this case, with the interviewee also reporting the project's lack of relevance to the organisation and strong dissatisfaction with the working group dynamics.

\section{Stage 2: Defining}

In the second stage, Defining, each organisation worked to more clearly articulate the nature of its chosen project challenge, recognising a unique set of issues and problems. The nature of these issues depended on the organisation's function (for example, whether it was policy-focused or operationally-focused), its disciplinary context, and its chosen focus area. As issues to do with implementing a MfO knowledge framework started to become clearer, the need for new, discipline-specific, knowledge was often recognised. In some cases, the need for a formal research effort was also identified. During this stage, organisations began to apply a set of conceptual models and procedural tools called "building blocks" that were undergoing concurrent, iterative, development by an inter-organisational working group. The building blocks are an example of boundary objects, objects that embody knowledge and able to be interpreted in different ways owing to their interpretive flexibility, thus facilitating knowledge sharing (Carlile \& Rebentisch, 2003; Pawlowski \& Robey, 2004).

The key issues identified during the Defining stage were based around the problem of how to apply and adapt the MfO knowledge framework and tools to each organisation's context. Adapting the new knowledge was difficult and required translation and interpretation

Organisational issues that arose during the Defining stage ranged from high level questions concerning the MfO knowledge framework (e.g.: "What should our key outcomes be?", "How can we measure them at a national level?", and "What sorts of interventions would we make to achieve the desired outcomes?"), to more practically focused concerns (e.g.: "How can we measure the effect of interventions on outcomes?" and "How can we work with our external partners towards a key outcome?".) In general, those organisations without prior experience in applying the framework, and/or with a poor perceived fit between MfO and their traditional discipline, identified higher-level issues than those without such experience.

\section{Key factors affecting knowledge transfer}

The organisational factors outlined in the previous stage continued to underpin the knowledge transfer project, but do not appear to have played such a key part during this stage. 


\section{(a) Inaccessible language}

All of the participants identified the abstract, theoretical, and technical nature of the language associated with MfO as being an impediment to the transfer of knowledge. MfO language created difficulties in three respects: firstly, it was seen as abstract and conceptual, making it inaccessible and difficult to move "from ideas to activity"; secondly, it was considered to be lacking in relevance to workers outside of the policy area; and thirdly, some of the MfO terminology was similar, or identical, to existing organisational language, leading to confusion regarding its intended meaning.

One participant described the degree of irrelevance MfO language presented for an 'average manager':

"I was very concerned at the beginning that what was coming out ..was supposed to be guidance for the whole public sector (but) would be unintelligible to your average manager...I said 'This is all very well, but Joe Bloggs who sits managing a very busy office in Auckland's North Shore can't understand a word of what you're talking about, so we've got to talk about it in terms that people can understand"'.

Another participant noted that people in their organisation commonly confused outcomes (a MfO concept), with outputs and/or inputs (existing strategic terms).

The technical term intervention logic, a key part of the MfO model, was mentioned as being particularly difficult by two participants.

"Things like 'intervention logic' ... people kind of think "oh, what's that?"

The above issues relating to language illustrate Carlile and Rbentisch's (2003) findings about how knowledge representation impacts on knowledge transfer: "As knowledge becomes more highly specialized, it develops its own terminology; nomenclature... which typically reside with specialist... but (this) by definition restricts the accessibility of the knowledge to the novice (p.1189)." However, these authors do also acknowledge the usefulness of shared language (as well as shared methods) in promoting knowledge transfer across specialised domains.

In order to address the problems associated with the accessibility of language, participants reported needing to undertake substantial translation and interpretation efforts.

\section{(b) Non-transferable examples}

A related problem, noted by several participants, was the use of case studies and examples to illustrate MfO in practice that could not be readily transferred to other organisational contexts. These examples, provided by project stakeholders, were not only from 'foreign' disciplines, but were also from organisations that had an operational focus, and so could not be transferred into policy-focused organisations. In addition, they were seen as being simple models, unable to be transferred to a more complex context.

"The models that were held up...were very good for some sets of people, but if you're working in a policy industry, they're no good at all...(If you're) delivering a service where you can see a result at the end of it - be it a dead bod or a prisoner who hasn't re-offended, or 
something that you can see - it's much simpler than trying to establish that, for example, this wonderful (piece of policy) analysis that you've done has actually added value in terms of the actual deliverable for a New Zealander out there."

The problem of non-transferability equates with a recognised constraint in translation theory; the problem of 'lack of equivalence' (the absence of corresponding words or concepts), outlined by Holden and Kortzfleisch (2004). It also supports Carlile \& Rebentisch's (2003)finding that "knowledge developed within different practices constrains or shapes action, value, and choice along different pathways" (p. 1189).

\section{(c) Boundary objects (Conceptual models and procedural tools)}

The Working Group developed a set of step-by-step models and tools specifically for implementing MfO, and to map out the MfO process and 'MfO cycle'. These tools were called "building blocks", and were used by participating organisations and modified by the Working Group as the project progressed. Most participants saw these building blocks as being valuable, with the usefulness of individual tools varying according to the amount of MfO experience and knowledge held by organisations:

"We were coming from a long way behind... The initial building blocks...were easy to bring into the organisation because (they reflected out state of development...but some of the other building blocks are beyond where we are at the moment."

The Pathfinder building blocks are an example of boundary objects (Carlile \& Rebentisch, 2003; Pawlowski \& Robey, 2004). Boundary objects are ways of representing knowledge, including shared language, shared methods and processes, people such as technical experts, and shareable artefacts; that can facilitate the translation and transformation of knowledge across different domains, owing to their interpretive flexibility.

Boundary objects in the form of the building blocks were seen by participants as being more transferable than the discipline-specific case studies used within the Pathfinder Working Group (PWG). Some participants reported using familiar strategic concepts, such as benchmarking and best practice, in conjunction with the building blocks, to help clarify knowledge goals, enabling the MfO initiative to fit in with what one participant described as "already-in-place existing thought methods."

\section{Stage 3: Seeking}

During the third stage, Seeking, organisations began actively seeking solutions and acquiring new knowledge to help them solve the issues that they had identified. This included searching for explicit knowledge, such as data to enable the linking of planned interventions with measurable outcomes, searching for tacit knowledge, such as knowledge to do with processes and problem-solving, and synthesising; and interpreting the knowledge that had been gathered.

This stage was characterised by a high degree of socialisation and knowledge sharing, with particular value coming from intra-organisational networking. 
Knowledge was sought within the organisation, and also from partner organisations (discipline related knowledge), and from members of the PWG (knowledge to do with strategy and processes). In general, there were limited external sources of knowledge to do with MfO, so the need to create new knowledge internally was high.

\section{Key factors affecting knowledge transfer}

Analysis of interviews identified both the following factors affecting knowledge transfer during the Seeking stage.

\section{(a) Lack of relevant discipline-specific knowledge}

The key problem faced by organisations during the Seeking stage was a lack of relevant knowledge, information, and experience both within their own organisation and within partner organisations from their discipline area. This problem was mentioned by five of the seven participants. This lack of knowledge applied both to international 'peers' (organisations in the same discipline area) and to national 'partners' (organisations that would eventually need to work with the initiating organisation in generating its outcomes) from whom knowledge was sought.

Participants mentioned the unavailability of the following types of information as being problematic: measurement tools for use at a national level, benchmarking information, tools to assist with prioritisation, and raw statistical data. For one participant, the absence of relevant discipline-based information amounted to an almost total vacuum:

"We're seen worldwide as being one of the leading departments in (our area). And when you recognise that the key outcomes that we're trying to achieve and the way you measure them are not well recognised worldwide, and there's no benchmarking worldwide, it's very, very hard to actually come up with the appropriate statement which says what you're trying to do, and then the method to measure it (with) and (how to) report against it, and to show that you've actually selected the right outputs."

Another participant described a failed attempt at replicating an example of MfO process from within their organisation (non-transferability within the organisation), arising from a lack of relevant quantitative data. This led to an increased awareness of the kind of difficulties faced by other organisations.

"Basically, we were struggling for information and useful data. It was interesting in that it was reaffirming the difficulties that other organisations have, because we could start seeing the difficulties ourselves...we were dealing with data that was difficult to transform into rigorous quantifiable amounts."

In this case, a total lack of experience with MfO by the organisation's State sector partners provided an even more major obstacle, affecting buy-in, resulting in what the gatekeeper ${ }^{2}$ described as a "denial of responsibility" for the concept of outcomes, and an effective refusal

\footnotetext{
${ }^{2}$ A gatekeeper is a person in an organisation who is exposed to and who monitors external information that may or may not be relevant for the organisation's innovation activities (T. Allen, 1967; Katz \& Tushman, 1981). The gatekeeper therefore spans a knowledge boundary between an organisation and its environment. Because their role involves ascertaining the relevance of external knowledge to the organisation, they are effectively tasked with controlling a 'knowledge gateway' to the organisation.
} 
to co-operate in the project This was problematic, given that the MfO model promotes the need for joint ownership of outcomes. The response was to seek the involvement of external experts.

During the latter part of the Seeking stage, some organisations began to compensate for the lack of existing information by building up an information base or database. Creating an information base was seen as valuable for its potential to support future MfO work:

"What I realise now is that (we were)... building up the data, the base of data and information from which further analysis will be able to shed further light about how to go on improving (for delivering our key outcome)"

\section{(b) Nature of team approach}

The Seeking stage (and also the stage that followed, Articulating), involved a limited number of people from each organisation in seeking and sharing new knowledge (and later in articulating new knowledge). Of the six participants who had reached this stage, five outlined how their organisation had adopted a "core team approach" (with 5-13 staff being closely involved in the project, working with others across the organisation as required). The gatekeeper was the central person involved in the core group, and was supported by a senior manager, usually a member of the project sponsors' group.

One participant described involving of a group beyond the core team, comprising senior staff representing each business unit. Others outlined involving key individuals from the business in a less formal capacity:

"There was a bit of an educative process with some of the key people in the operational business groups. We saw that...if they saw what was happening and could be involved and learn things, it was the best chance of getting the business units to move towards more (of an) outcomes rather than outputs focus."

One participant reported using a different model, a series of "key step" teams led by senior managers. This approach was undertaken in order to force ownership of the new knowledge by senior managers, but resulted in the gatekeeper being the sole source of continuity across the organisation.

It is probably no co-incidence that the participant who described using this approach also reported an instance of misapplication of MfO.

"There were papers circulated to those key step teams, and there was training provided... It didn't prevent people from wanting to manipulate the process to meet their own requirements"

This participant suggested that in future, a composite approach, involving a core team working across the business together with some business unit ownership, might be more effective:

"The key step process worked for us, but...it never got probably the right people engaged.... There are two options: you either get management significantly involved and get (them) to drive it, which is what we tried to do, or you have a small team that are passionate about performance measures and outcomes and out puts and fully understand it, and then use them as a team to actually go out and look across the business....it's a case of which one's 
going to be more useful ... We were forcing the ownership process, in terms of getting general managers, and other managers at the next levels, owning the need to have really good performance measures in place, which we needed to manage the business, which would also provide us with external information to meet the central agencies' requirements. (But) I think l'd go back and revisit that process again, and it might be a matrix or mix between the two that we might end up with."

\section{Stage 4: Articulating}

In the fourth stage, Articulating, each organisation articulated and formalised solutions, transforming their newly acquired or created knowledge into explicit knowledge artefacts, such as Statements of Intent (Sols) ${ }^{3}$,. Some organisations also wrote case studies, in order to demonstrate the applications of the new knowledge framework (such as the application of "intervention logic") for future learning in the inter-organisational context.

The Articulating stage involved adapting and codifying the knowledge gathered from internal and external sources, with further use of the conceptual tools (boundary objects called 'building blocks') developed by the PWG.

In general, organisations documented knowledge in an iterative fashion during this stage, allowing for feedback from within the organisation while the participant shared knowledge and sought feedback from the other PWG members. The need for Sols to be revised annually provided an opportunity for higher level iterative application of MfO ideas.

The Articulating stage corresponds closely with the Externalisation stage of Nonaka's SECI model (ibid), in which companies document and formalise new knowledge that has been previously developed through the processes of socialisation.

\section{Key factors affecting knowledge transfer}

Knowledge transfer during the Articulating stage was facilitated through the process of codification (creating new strategic documents, or organisational boundary objects, to capture emergent knowledge) and the use of existing inter-organisational boundary objects; the conceptual and procedural 'building blocks' described in a previous section, to guide the codification process.

\section{(a) Codification - creation of strategic documents}

The Articulation phase stage involved codifying new MfO knowledge in the form of strategic documents (or knowledge artefacts) with each organisation's key output being a Statement of Intent (Sol).

"(I was) feeding my experiences and information from the (Working) Group into our Sol development and forecast."

The creation of a Sol was a knowledge-focusing activity, resulting in a high-level snap-shot that captured the organisation's current state of MfO knowledge. The Sol would function during the next stage as a boundary object for use within the organisation.

\footnotetext{
${ }^{3}$ These are documents that carry the essence of MfO intent and identify key strategic outcomes.
} 
Participants viewed the process and experience of creating the Sol as being valuable in its own right, enforcing focused and strategic thinking:

"In terms of producing our Statement of Intent, our organisation's done much more in terms of strategic planning (than it had done previously)."

One participant reported discovering the need to simplify their organisation's outcomes framework as a result of developing the Sol. This resulted in a decision to reduce the number of organisational outcomes so that the Sol had a clearer high-level focus and so it would enable business units to work together more easily at a strategic level.

The fact that a new Sol was required on an annual basis was seen by participants as beneficial, enabling iterative improvements. (In the words of one gatekeeper: It "can be born again each time.") During the two-year period of the project, two Sols were developed by participating organisations:

"It was very iterative and that was reflected in the Sol process...The first year was, sort of, go (number) one, and then the second year you had another go and sharpened it".

The capturing and documentation of knowledge in the Sol, a form that could be readily and that had high level status, was also critical for enabling the next stages (Integrating and Disseminating) to occur.

\section{(b) Boundary objects (conceptual models and procedural tools)}

The Articulating stage required organisations to codify and further adapt knowledge that had been gathered and synthesised from internal and external sources. This was facilitated by the further use of boundary objects, the conceptual models or tools (called 'building blocks') that were developed by the PWG. In the absence of relevant, convertible, examples of Sols (reported by one participant), the use of a generic conceptual model for the new Sol was seen as particularly useful, enforcing a discipline to the codification of knowledge. (Only one person described the building blocks as not being useful, but this participant's organisation undertook passive involvement and did not proceed beyond the Engaging stage.)

The source of perceived value in the Pathfinder building blocks was their generic nature, the fact that they were specifically designed for use in association with $\mathrm{MfO}$, and the fact that they had been 'field tested' by different organisations.

One participant reported, however, that the building blocks were not universally accepted by others within the organisation:

"A lot of people in the (organisation) are passionate about what they do... Sometimes, even if you have a good set of templates or processes,...it doesn't necessarily mean that they'll follow them."

Despite this, they were seen by the same participant as having provided a useful catalyst for discussion:

"...and it's been a catalyst as well, in terms of discussion - because there have been some disagreements within (the organisation) about whether all the tools are necessary." 
The building blocks were refined in the second year of the project, providing a new challenge, in the form of a discontinuity of knowledge between the original gatekeepers (who by now had extensive experience with MfO), and newer members of the PWG, from organisations that were new to the project.

"After a year (the tools and guidance documents) required going back and revamping because you had moved on, and this was one of the challenges because the group that had been doing outcome based performance management for the whole period; their experiences no longer matched the experiences of the organisations who weren't doing it, so we ended up having overviews or summaries that were for the getting started phase...There was that tension between teaching those people who were already doing it whereas people at the beginning of the process did need that information."

\section{Stage 5: Integrating}

During the fifth stage of knowledge transfer, Integrating, organisations worked to integrate the newly articulated MfO knowledge with their existing strategic and managerial knowledge. This stage happened concurrently with the sixth stage, as the newly created Sols were disseminated throughout the organisation. (Some organisations focused exclusively on dissemination, as there was no requirement for integration prior to MfO becoming mandatory for the sector.)

During the Integrating phase, the MfO framework began to be used for strategic planning at the senior management and business unit level, with the newly created Sols providing a guiding framework. The language of MfO began to be adopted by managers, strategy and policy-makers within the organisation, and the PWG tools were adapted and applied to new organisational contexts.

Organisations identified new issues relating to the introduction of $\mathrm{MfO}$, often based around a lack of congruence between the high level outcomes identified in their new Sols and their traditional framework of outputs. New focal areas for the application of MfO were identified, and the process of Defining (Stage 2) began afresh.

The Integrating stage can be seen as broadly equivalent to the combination stage of Nonaka's SECI model, where communication across organisational groups becomes critical in order to achieve wider cross-organisational knowledge transfer.

\section{Key factors affecting knowledge transfer}

A number of factors were identified by participants as contributing to knowledge transfer during this stage. Some of these are the same as those outlined in previous stages and so will not be recapped upon (i.e., the support of the CEO, gatekeeper networking, the gatekeeper's role as a translator, and the use of conceptual models and tools). The key factors that are unique to the Integrating stage, and/or that began to impact on knowledge transfer during this stage, are outlined below. 


\section{(a) Project Framework}

Several participants mentioned the project framework of Pathfinder, with its requirement to deliver results, as being a key factor in improving their strategic planning approach and organisational systems:

"Our planning process stepped up a gear...These sorts of things end up reinforcing principles of good management... We were getting the most significant things done through our external partners...And what we were seeking to do with the corporate planning stuff was to start turning the blowtorch which we'd applied to the issue externally... turning some of that analytical attention internally"

\section{(b) Type of team approach}

The problems of integrating new organisational knowledge appear to have been exacerbated by the adoption of a "key step team" approach (described above). This resulted in early complexity of knowledge and a need for simplification:

"Because we had seven key step teams, not all of them worked the same way, even though they have the same instructions and the same training...And so the integration of the seven key steps into what the department was doing wasn't particularly clear.. That was the thing that drove the desire to reduce the number of key outcomes down to two so that they could be integrated better, so that people actually understood what their component of it was."

Two participants used the metaphor of a road, or road map, with the key organisational outcome equating with a destination point, to describe the risk of losing focus mid-journey, and the resulting complexity that could arise:

"You can have a road map for getting to Wellington, and there are a hundred different ways (to get there), but the objective is still to get to Wellington..(That's what) we were losing sight of. A lot of our objectives were to get to the New Plymouths and the Wanganuis on the way to Wellington. So we've tried to focus (on the destination) a lot more."

\section{(c) Ownership by senior managers}

Active ownership of the new MfO knowledge framework by senior managers was described by three participants as being essential for the integration of $\mathrm{MfO}$, but was not seen as an easy thing to achieve. One participant described gaining such high-level ownership as a "huge achievement," while another described their approach as "forcing the ownership process". A third gatekeeper emphasised the need for senior managers to engage in difficult decision-making and prioritisation exercises as part of the knowledge integration task:

"It re-emphasised the importance of senior management. You know, the chief executive commitment stuff... being prepared to have quite tough decisions and (have) prioritisation issues on the table... If you're going into this sort of thing you've got to be prepared to deal with some of this stuff and it's not easy. It forces different parts of the organisation to think about things in ways in which they just hadn't before..It can shed real light for more enlightened managers." 


\section{(d) Traditional structures}

The traditional organisational structures of business units and the associated 'silo-based' thinking created a barrier to integration of $\mathrm{MfO}$, particularly in cases where two or more business units with unrelated outputs foci needed to contribute to a single outcome. The problem of silo-based thinking mitigates against what Reid et al (2001) describes as combinative capacity. Two participants commented that the approach of driving MfO directly down from the Sol into business units was problematic due to the existing structures, and one said that a matrix type approach would be preferable, given the "many-to-many linkages" that were needed.

This situation was compounded by a degree of resistance to change: One gatekeeper reported that some of their colleagues had viewed the MfO work as a "flavour of the month" exercise, while another described a case of intentional misapplication of the MfO model, with "people wanting to manipulate some of the process to meet their own requirements".

As a result of issues relating to organisational structure that arose during the Integrating stage, two participants reported realising the need for a change management process in association with implementing MfO. One participant described how, as Integrating occurred, a strategy group with no relationship to organisational outcomes was found to have outlived its usefulness. Another outlined the need for introducing new staff performance management systems that supported MfO.

\section{(e) Appropriation and simplification of language}

Participants described the repeated and consistent use of appropriated MfO terminology (as adopted in the Sol), and the simplification of MfO language as enabling deeper integration of MfO knowledge. Total consistency in how language associated with the new knowledge was used was therefore essential:

"You just keep using the words the way you mean it and eventually that meaning comes through."

\section{(f) Boundary objects (Sols and case studies)}

All the participants described the Sol (produced during the previous stage) as being very useful, emphasising the importance of the new MfO knowledge, and promoting its integration. Equally importantly, the Sol was valued by participants for providing a formal reference framework within which the ongoing MfO work needed to occur. It acted as a boundary object, ensuring widespread visibility of the new knowledge framework, acting as a central point of reference for people within and outside of the organisation. For the gatekeepers and developers of organisational strategy, the first 'MfO-focused' Sol acted as a useful 'stake in the ground' while the relatively new MfO concept could continue to be integrated and to evolve.

"It's ...been about trying to map what our business is about, and then being able to use that map to say, well, okay, that's the destination we're trying to get to." 
Formal workshops about MfO were run by the Working Group for senior managers. These featured a combination of models and examples, which were seen as a useful way of engaging senior staff and conveying relevance.

\section{Stage 6: Disseminating}

In the sixth and final stage, Disseminating, organisations actively disseminated their new MfO knowledge to staff at various levels throughout business units, and (if relevant) operational arms and regional centres. This stage involved a concerted effort and multiple communication methods: informal socialisation to share knowledge (such as manager-to-staff interactions) and more formalised communication of knowledge (such as workshops and the distribution of documents and other artefacts). Distribution and explanation of the new Sol, with supporting material, was central to this process.

The issue of buy-in was faced by all organisations to a varying degree during the Disseminating stage, as individuals or groups struggled to make sense of the new model, or felt threatened by it. Several participants reported the need to translate and interpret the new Sol for individuals within their organisation, in order to address the question, "how does this relate to my job?". During this stage, policy-based organisations faced the additional challenge of distributing knowledge beyond their organisation to third parties within the State Sector that carry out an implementation role. In order to underline the strategic importance of the MfO concept, the CEO and senior managers became involved in presenting the Sol, in some cases undertaking regional tours to promote it. A practice involving "trickle-down" of the new knowledge was also undertaken.

\section{Key factors affecting knowledge transfer}

The following factors impacted on knowledge transfer during the Disseminating stage.

\section{(a) Distribution of staff}

One participant described a wide geographic distribution of staff as a barrier to knowledge transfer.

"We're a widely distributed organisation and secondly most of the staff are passionate about what they do...In some cases that passion turns into personal projects that they want to do rather than what the department might want to do... Those things cause some difficulty in terms of being able to get a common view."

\section{(b) Subcultures}

A workplace culture based around personal passions was identified by the participant referred to above as a barrier to reaching a common state of organisational knowledge. This supports Long and Fahey's (2000) finding (cited by Karlsen and Gottschalk (2004) that "Culture - and subcultures in particular - shape assumptions about what knowledge is worth exchanging and...the processes by which new knowledge - with its accompanying uncertainties - is created, legitimated, and distributed in organizations" (p.4) 
A key aspect of knowledge transfer is "absorptive capacity", the readiness and willingness to recognise the value of new knowledge, absorb it and apply it (Rolland et al., 2003). Most studies of absorptive capacity are focused at the level of firms or business units (European Business Forum, 2004). However, a culture of personal passions can impact on absorptive capacity at an individual level.

\section{(c) "Trickle-down"}

Three participants outlined how their organisations adopted a strategic "trickle-down" approach to MfO knowledge dissemination, with the aim of ensuring that senior staff would gain ownership of MfO and assist with the dissemination task:

"(Our staff will typically) take the words as meaning something different, even the definitions they'll take as meaning something different. So it's quite hard for us...to get absolute clarity about what we're trying to do. The only way we can do that is by inculcating it right down through the management chain and getting the managers to understand it and filtering it down".

"Trickle-down" resulted in managers incorporating MfO knowledge in their training programmes and divisional policy development:

"It was done in layers - the managers, those involved in setting goals and assigning workwe worked with them for the outcomes focus and then it trickles down to their staff in what those goals are and what they need and the training they receive So it's built into training, it's built into the practice statements, it's built into the policies...."

\section{(d) Face-to-face communication}

Participants described a range of face-to-face communication methods undertaken by their organisation as being useful at this stage, including formal workshops, meetings, MfO timeslots in existing operational meetings, and training sessions. In one case, the CEO undertook extensive regional tours to speak directly to remotely located staff, helping underline the importance of the new knowledge. Storytelling and an iterative approach to dissemination (repeated "doses" of knowledge) were part of the face-to-face communication strategies.

\section{(e) Distribution of codified knowledge}

The value of published, or codified, knowledge (in the form of the published Sol and associated documentation) was high at this stage, with documents being distributed to staff for use as a reference document.

"Our Sol is actually quite a well-read document, whereas if we go back five years it never was... Now we run off two thousand copies and most staff members have access to a Sol. So they know that it describes the work they're doing, and somewhere in the Sol they will find the work they carry out."

One participant described distributing a three-dimensional pyramid showing how people's roles linked with key outcomes, and outlined the usefulness of this prop in subliminally reinforcing MfO messages: 
"Everyone was given one for their desks...lt was good because it (MfO thinking) got in without people realising it was getting in."

Surprisingly, only one participant mentioned the use of the intranet as a key tool for disseminating codified MfO knowledge to staff.

"There's an intranet (where) you can look at a whole lot of information. There's regular items on particular topics. There's been a lot of dissemination (of information about MfO) through the computer. All of the documents are accessible; people no matter where they are can just put in a number and the document will come up."

It should be noted that the forms of knowledge codification described above were used to complement a high level of face-to-face communication (workshops, meetings, presentations etc) and their usefulness cannot be divorced from these more social forms of communication.

\section{DISCUSSION AND IMPLICATIONS}

The organisational factors discussed in the previous sections are consistent with two existing models for the role of organisations in knowledge transfer (both developed in the setting of learning-based alliances): These are Reid et al's (2001) five key capabilities: experience, resources, combinative capacity, absorptive capacity, and firm design; and Rolland and Chauvel's (2003) four initial structural conditions (strategic intent, organisational culture, trust, and organisational form) and two dependent variables (transparency and learning, or absorptive, capacity). However, the issue of fit between new knowledge and an organisation's discipline area - which was a significant factor in this study - is not catered for by either of these models, and does not appear to have been considered elsewhere in the knowledge transfer literature.

A number of implications arise from this study for practitioners and researchers. Firstly, an issue relevant to practitioners is the finding that organisations recognised the extent of challenge posed by new knowledge at an early stage. The implication of this is that if there is an early recognised issue to do with lack of 'fit' of new knowledge, it can reasonably be assumed that the process of transfer will be difficult, and so the opportunity arises at this time to plan for appropriate resourcing and skill-sets. Of particular importance in the situation of low fit are the skills of translation and interpretation. Appointment of a dedicated researcher is also likely to be beneficial in cases where there is a lack of existing information or data to support organisational application of new knowledge. The gatekeeper should also be encouraged to undertake networking with experienced colleagues who work in a similar field in other organisations.

Secondly, there is a need for CEOs and senior managers to recognise the complex nature of inter-organisational knowledge transfer and support it accordingly. In this study, organisations worked to adopt an emerging knowledge framework that originated outside of their organisation. In the process, they found that they needed to seek solutions about how to implement this knowledge from multiple knowledge sources, both within and outside of their organisations, and internationally. This process required both formal and informal 
communication methods, and was supported by a mix of tacit knowledge and explicit knowledge, information and data. Solutions were manufactured by combining and reinterpreting these multiple forms of knowledge, and by applying generic tools and processes. At different stages of this process, different factors operated as enablers of knowledge transfer.

Thirdly, an issue that arose in this study, and does not appear to be discussed elsewhere, is that the perceived degree of fit between new knowledge and an organisation's discipline may impact on absorptive capacity. While this appears to be logical, it should be further tested, with the aim of identifying ways to address the problem of fit.

Finally, previous researchers have identified boundary objects as useful for knowledge transfer, due to their ability to be interpreted in different ways (Carlile \& Rebentisch, 2003; Pawlowski \& Robey, 2004). Such objects appear to offer particular potential in an Inter-organisational context. A study to investigate the properties of successful and unsuccessful boundary objects (such as the type used in Pathfinder) in an inter-organisational context would add further value to the research literature. 


\section{REFERENCES}

Advisory Group for the Review of Centre. (2001). Report of the Advisory Group on the Review of the Centre: Wellington: State Services Commission.

Allen, N., \& Leeuwen, M. (2003). European Guide to Good Practice in Knowledge Management. Working Draft version 6.0. Retrieved 2003-08-09, from http://www.knowledgeboard.com/cgi-bin/.

Allen, T. (1967). Communications in the Research and Development Laboratory. Technology Review, 70, 31-37.

Benbasat, I., Goldstein, D., \& Mea, M. (1987). The case research strategy in studies of information systems. MIS Quarterly, 11, 368-386.

Betancourt, E. (1997). A New Approach to Developing Institutional Structure. Studies in International Development, 32(2), 3-28.

Carlile, P., \& Rebentisch, E. (2003). Into the Black Box: The Knowledge Transformation Cycle. Management Science, 49(9), 1180-1195.

Davenport, T., \& Prusak, L. (1998). Working Knowledge: how organizations manage what they know. Boston: Harvard Business School Press.

Davidson, C. V., P. (2002). Knowledge management: An introduction to creating competitive advantage from intellectual capital. Auckland: Tandem Press.

Earl, M. (1998). What on Earth is a CKO? IBM Survey, London Business School. Cited in Grant, R. (2000).

European Business Forum. (2004). Absorbing Knowledge. (Review of a paper by Pedersen, T, and others entitled "MNC Knowledge Transfer, Subsidiary Absorptive Capacity and HRM" from the Journal of International Business Studies; reference incomplete). European Business Forum, 17(55).

Gorgoglione, M. (2003). Strategies for Knowledge Transfer. Retrieved 2004-10-24, from http://www.knowledgeboard.com/cgi-bin/item.cgi?id=110132\&d=1\&h=417\&f=418\&da teformat $=\%$ o\% $20 \% \mathrm{~B} \% 20 \% \mathrm{Y}$.

Harada, T. (2003). Three steps in knowledge communication: the emergence of knowledge transformers. Research Policy, 32(10), 1737.

Hedlund, G. (1994). A Model of Knowledge Management and the N-form Corporation. Strategic Management Journal, 15, 73-90.

Hildreth, P., Kimble, C., \& Wright, P. (1998). Computer Mediated Communications and Communities of Practice, Proceedings of Ethicomp '98 (pp. 275-286). Netherlands: Erasmus University.

Holden, N., \& Von Kortzfleisch, H. (2004). Why cross-cultural knowledge transfer is a form of translation in more ways than you think. Knowledge and Process Management, 11(2), 127-137.

Husted, K., \& Michailova, S. (2003). Diagnosing and Fighting Knowledge-Sharing Hostility. Organizational Dynamics, 3(1), 60-73.

Karlsen, J., \& Gottschalk, P. (2004). Factors Affecting Knowledge Transfer in IT Projects. Engineering Management Journal, 16(1), 3-10.

Katz, R., \& Tushman, M. (1981). An Investigation into the Managerial Roles and Career Paths of Gatekeeper and Project Supervisors in a major R\&D Facility. $R \& D$ Management, 11, 103-110.

Lee, L., \& Al-Hawamdeh, S. (2002). Factors impacting knowledge sharing. Journal of Information and Knowledge Management, 1(1), 49-56.

Liebowitz, J. (2002). Facilitating innovation through knowledge sharing: A Look at the US Naval Surface Warfare Centre - Carderock Division. The Journal of Computer Information Systems, 45(5).

Loebecke, C., van Fenema, P., \& Powell, P. (1999). Co-opetition and knowledge transfer. The DATABASE for Advances in Information Systems, 30(2), 14-25. 
Nonaka, I. (1998). The Knowledge-Creating Company. Harvard Business Review on Knowledge Management.

Pathfinder. (2003). Pathfinder Website. Retrieved 2003-09-28, from http://io.ssc.govt.nz/pathfinder/.

Pawlowski, S., \& Robey, D. (2004). Bridging User Organizations: Knowledge Brokering and the Work of Information Technology Professionals. MIS Quarterly, 28(4), 645-672.

Reid, D., Bussiere, D., \& Greenaway, K. (2001). Alliance Formation issues for knowledge-based enterprises. International Journal of Management Reviews, 3(1), 79-101.

Rolland, N., \& Chauvel, D. (2000). Knowledge Transfer in Strategic Alliances. In C. Despres \& D. Chauvel (Eds.), Knowledge Horizons: The Present and the Promise of Knowledge Management (pp. 225-236). Boston: Butterworth-Heinemann.

Rolland, N., Chauvel, D., \& Despres, C. (2003). Knowledge Transfer and Organisational Learning in Strategic Alliances: Unpublished paper provided by Nicolas Rolland.

Scmetz, F. (2002). Introduction to KM in the Public Sector. Retrieved 2003-08-12, from http://www.knowledgeboard.com/cgi-bin/.

Snowden, D. (1999). Creating a Sustainable Knowledge Programme. Paper presented at the Optimizing Knowledge Management conference, London.

Waits, M. (2000). The added value of the industry cluster approach to economic analysis, strategy development, and service delivery. Economic Development Quarterly, 14(1), $35-50$.

Wenger, E. (2000). Communities of Practice. In C. Despres \& D. Chauvel (Eds.), Knowledge Horizons: The Present and the Promise of Knowledge Management (pp. 205-224). Boston: Butterworth-Heinemann.

Wright, A., \& de Joux, V. (2003). Case Studies of Innovation in the Public Service. Retrieved 2003-08-04, from http://www.treasury.govt.nz/innovation/default.asp.

Yin, R. (1994). Case Study Research: Designs and Methods (2nd ed.). California: Sage Publications.

Yoong, P. M., M. (2003). Sharing in a Co-opetative Environment: The Case of Business Clusters. Paper presented at the 7th Pacific Asia Conference on Information Systems, 10-13 July 2003, Adelaide, South Australia. 